\title{
Dynamical Behaviors of Rumor Spreading Model with Control Measures
}

\author{
Xia-Xia Zhao ${ }^{1,2}$ and Jian-Zhong Wang ${ }^{1,2}$ \\ ${ }^{1}$ National Key Laboratory for Electronic Measurement Technology, North University of China, Taiyuan, Shanxi 030051, China \\ ${ }^{2}$ Key Laboratory of Instrumentation Science and Dynamic Measurement, North University of China, Ministry of Education, \\ Taiyuan, Shanxi 030051, China
}

Correspondence should be addressed to Xia-Xia Zhao; xiaxiazhao12@163.com

Received 16 April 2014; Accepted 12 May 2014; Published 1 June 2014

Academic Editor: Sanling Yuan

Copyright (C) 2014 X.-X. Zhao and J.-Z. Wang. This is an open access article distributed under the Creative Commons Attribution License, which permits unrestricted use, distribution, and reproduction in any medium, provided the original work is properly cited.

\begin{abstract}
Rumor has no basis in fact and flies around. And in general, it is propagated for a certain motivation, either for business, economy, or pleasure. It is found that the web does expose us to more rumor and increase the speed of the rumors spread. Corresponding to these new ways of spreading, the government should carry out some measures, such as issuing message by media, punishing the principal spreader, and enhancing management of the internet. In order to assess these measures, dynamical models without and with control measures are established. Firstly, for two models, equilibria and the basic reproduction number of models are discussed. More importantly, numerical simulation is implemented to assess control measures of rumor spread between individualsto-individuals and medium-to-individuals. Finally, it is found that the amount of message released by government has the greatest influence on the rumor spread. The reliability of government and the cognizance ability of the public are more important. Besides that, monitoring the internet to prevent the spread of rumor is more important than deleting messages in media which already existed. Moreover, when the minority of people are punished, the control effect is obvious.
\end{abstract}

\section{Introduction}

As a typical social phenomenon, rumor has no basis in fact and flies around, especially when major public events happen and people do not have exact information and knowledge about the events; the rumor is dispersed by some people for achieving the specific purpose. It has been described in detail by some pieces of literature [1-6]. In the modern society, the rumor not only has not disappeared but also, with the development of the communication transmission modes, such as internet, telephone, and advanced information technology, spreads more quickly and the scope involved is much broader. Thus, the internet rumors become an important factor that influences the current social harmony and stability in emergencies and all kinds of crisis, and it is becoming the focus of the netizens and governments at all levels.

The classical models to study the spread of rumor were given by Daley and Kendall and Maki and Thomson [7, 8]. Since the dissemination process of rumor is similar to the spreading of infectious disease, epidemic models have usually been applied to investigate the spread of rumors [9-13]. The ignorant, the spreader, and the stifler are equivalent to the susceptible, the infected, and the recovered. Some models are established based on network [14-19]. Some are built on the basis of the random theory [20-23].

With rapid development of today's society, besides propagation by word of mouth, rumors also can be spread by public homepage, SMS, e-mails, or blogging that provide faster velocity of transmission $[9,24,25]$. The new type of transmission mode has been studied dynamically by [26]. It established an ISRW dynamical system including spreading between individuals and medium-to-individuals to describe the actual pattern of transmission. With regard to the internet rumor, the government should share real information in a timely manner with the public to avoid the public hazard [27]. In 1953, the formula that describes the generation of rumor was proposed by Cross. $R=I \times a / c$, where $I$ is the importance of events, $a$ is ambiguity of events, and $c$ is the critical ability of the public. There are some models to assess the control 
TABLE 1: Description of parameters in the system (1).

\begin{tabular}{|c|c|c|c|}
\hline Parameters & Value & Unit & Comments \\
\hline $\bar{A}$ & $16000000 / 365$ & Day $^{-1}$ & The birth rate of human \\
\hline$\beta$ & $50 \times 0.005 / 1350000000$ & Day $^{-1}$ & $S(t)$-to- $I(t)$ transmission rate \\
\hline$\alpha$ & $10^{-10}$ & Day $^{-1}$ & $W(t)$-to- $I(t)$ transmission rate \\
\hline$d$ & $1 / 60 / 365$ & Day $^{-1}$ & The natural mortality rate of human \\
\hline$\theta$ & 0.5 & None & The rate of being $S(t)$ after transmission \\
\hline$m$ & 0.0001 & Day $^{-1}$ & The transformation rate of $S(t)$ into $R(t)$ \\
\hline$k$ & 1 & Day $^{-1}$ & The submerged rate of message \\
\hline$\lambda$ & $300 \times 0.00001$ & Day $^{-1}$ & The disseminating quantity of messages per spreader \\
\hline$\xi_{1}$ & $50 \times 0.0001 / 1350000000$ & Day $^{-1}$ & The transformation rate to $R(t)$ \\
\hline$\xi_{2}$ & $50 \times 0.0001 / 1350000000$ & Day $^{-1}$ & $R(t)$-to- $S(t)$ transmission rate \\
\hline$I(0)$ & $1350000000-1000000$ & Individual & The initial number of the susceptible individuals \\
\hline$S(0)$ & 1000000 & Individual & The initial number of the spreaders \\
\hline$R(0)$ & 0 & Individual & The initial number of the stiflers \\
\hline$W(0)$ & 0 & Individual & The initial quantity of messages by spreader \\
\hline
\end{tabular}

measures $[28,29]$. In order to control the rumor spreading, we can focus on the credibility of the authorities' media [30-32] and increasing the cognizance ability of the public. Besides, the government should give a certain punishment for the spreader. Therefore, this paper mainly assesses the effect of these measures.

\section{A Dynamical System for Rumor Spreading}

Without consideration of government measures, the dynamical system we establish will include the following four classes: the susceptible individual $(I)$, the spreader $(S)$, the stifler $(R)$, and the message in media $(W)$. Here, the bilinear incidence rate is considered. The interpretation of parameters can be seen in Table 1 . The model we employ is as follows:

$$
\begin{gathered}
\frac{d I(t)}{d t}=A-\beta I(t) S(t)-\alpha I(t) W(t)-d I(t), \\
\frac{d S(t)}{d t}=\theta \beta I(t) S(t)+\theta \alpha I(t) W(t)-2 \xi_{1} S^{2}(t) \\
\quad-m S(t)-\xi_{2} S(t) R(t)-d S(t), \\
\frac{d R(t)}{d t}=(1-\theta) \beta I(t) S(t)+(1-\theta) \alpha I(t) W(t)+2 \xi_{1} S^{2}(t) \\
+m S(t)+\xi_{2} S(t) R(t)-d R(t), \\
\frac{d W(t)}{d t}=\lambda S(t)-k W(t) .
\end{gathered}
$$

\section{Dynamical Behaviors of System (1)}

It is easy to know that $d N(t) / d t=A-d N$. So, the positive invariant set is $\Gamma=\{(I(t), S(t), R(t), W(t), G(t)) \mid I(t) \geq$ $0, S(t) \geq 0, R(t) \geq 0, W(t) \geq 0, G(t) \geq 0,0 \leq I(t)+S(t)+$ $R(t) \leq A / d\}$. The disease-free equilibrium is $E_{0}=\left(I^{0}, 0,0,0\right)$, where $I^{0}=A / d$.

The basic reproduction number, that is, the expected number of secondary spreaders produced by a spreader in a completely ignorant population [33-35], can be calculated as follows:

$$
R_{0}=\frac{\theta \beta I^{0}}{m+d}+\frac{\lambda \theta \alpha I^{0}}{(m+d) k}
$$

The detailed calculation method can be seen in [35] and the behavior of (1) is discussed in Theorem 1 .

Theorem 1. (a) When $R_{0}<1$, the disease-free equilibrium $E_{0}$ is globally asymptotically stable.

(b) When $R_{0}>1$, the disease-free equilibrium $E_{0}$ is unstable.

Proof. (a) Define a Lyapunov function

$$
L=k S+\theta \alpha I^{0} W \geq 0
$$

When $R_{0}<1$, the Lyapunov function satisfies

$$
\begin{aligned}
\dot{L}= & k\left[\theta \beta I(t) S(t)+\theta \alpha I(t) W(t)-2 \xi_{1} S^{2}(t)-m S(t)\right. \\
& \left.\quad-\xi_{2} S(t) R(t)-d S(t)\right] \\
& +\theta \alpha I^{0}[\lambda S(t)-k W(t)] \\
\leq & k \theta \beta I^{0} S+\theta \alpha \lambda I^{0} S-k(m+d) S \\
= & k S(m+d)\left[\frac{\theta \beta I^{0}}{m+d}+\frac{\lambda \theta \alpha I^{0}}{(m+d) k}-1\right] \\
= & k S(m+d)\left[R_{0}-1\right] \\
\leq & 0 .
\end{aligned}
$$

It is easy to know that $\dot{L}=0$ only hold when $S=0$. As a result, the disease-free equilibrium point $E_{0}$ is the only fixed point of the system. By applying the Lyapunov-LaSalle asymptotic stability theorem [36, 37], the disease-free quilibrium point $E_{0}$ is globally asymptotically stable. 


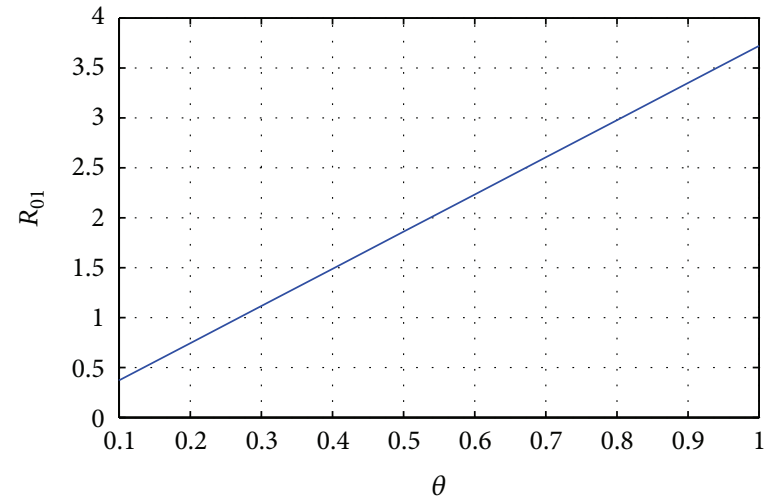

(a)

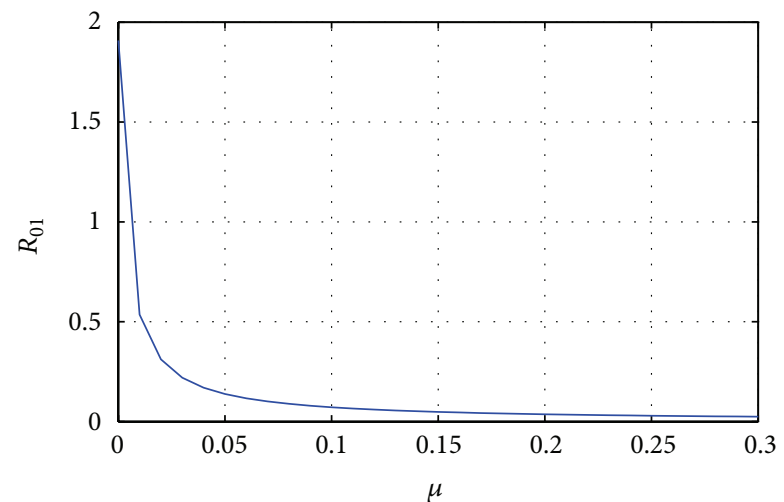

(c)

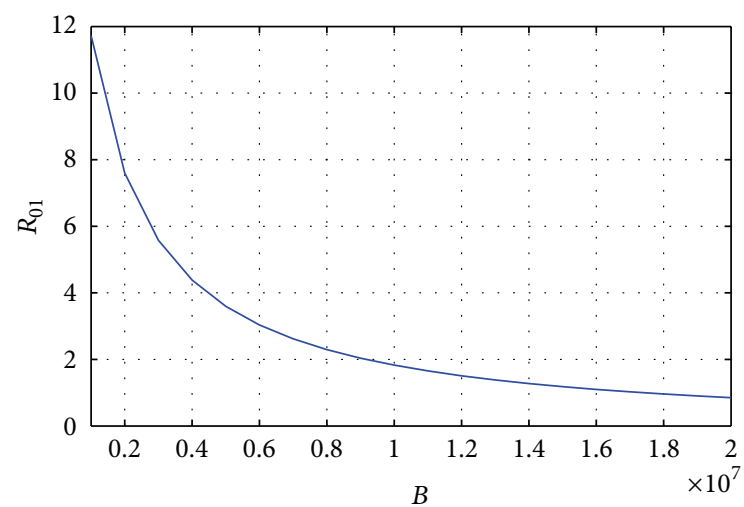

(e)

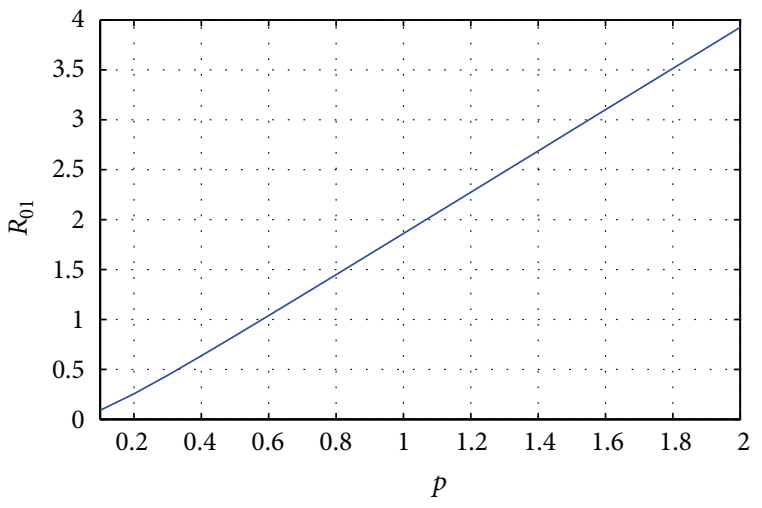

(g)

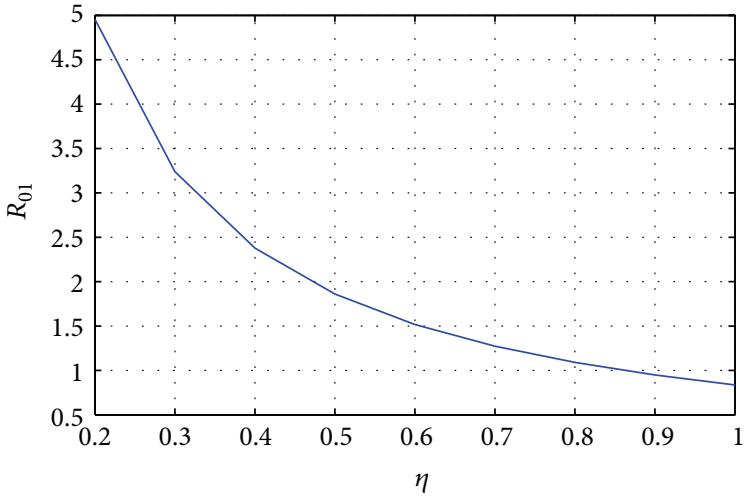

(b)

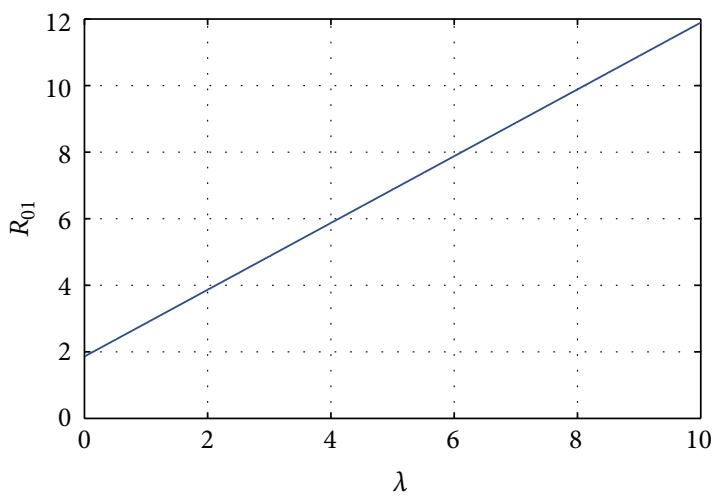

(d)

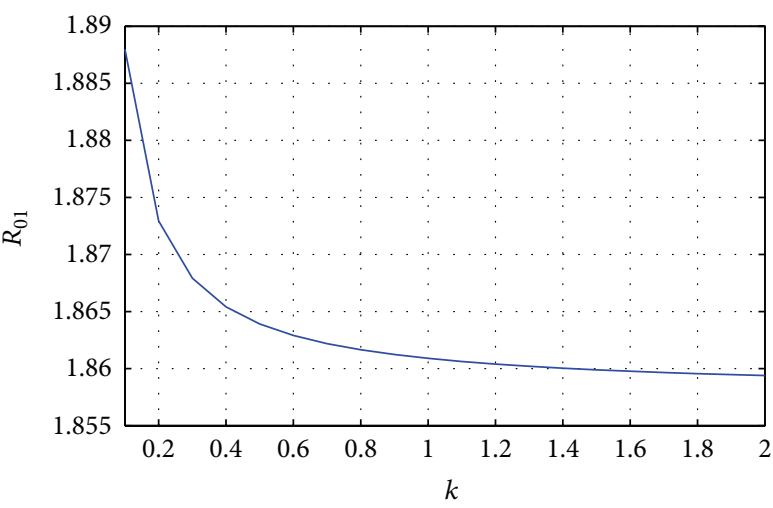

(f)

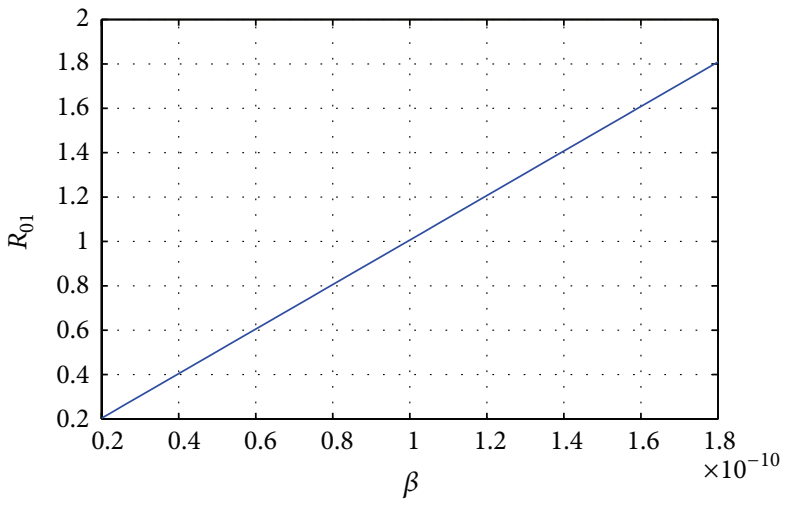

(h)

Figure 1: Continued. 


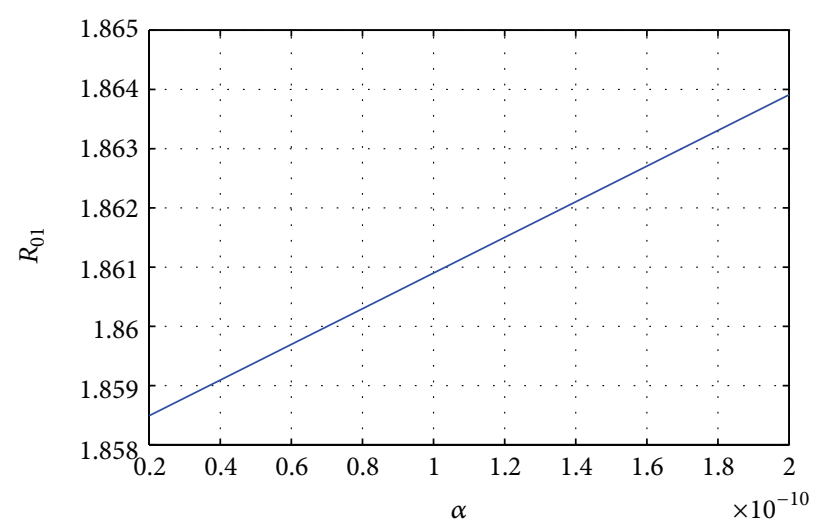

(i)

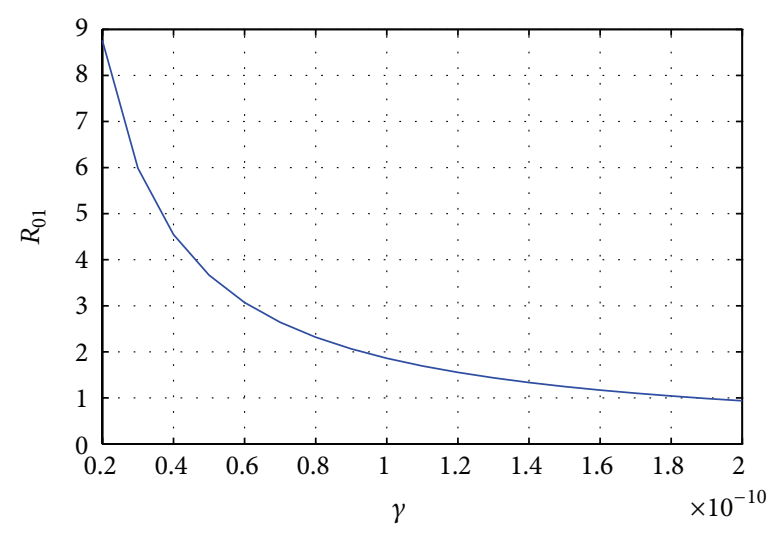

(j)

FIGURE 1: The sensitivity of $R_{01}$ in terms of parameters.

(b) The Jacobian matrix at the $E_{0}$ is the Jacobian

$$
\left.J\right|_{E_{0}}=\left(\begin{array}{cccc}
-d & -\beta I^{0} & 0 & -\alpha I^{0} \\
0 & \theta \beta I^{0}-(m+d) & 0 & \theta \alpha I^{0} \\
0 & (1-\theta) \beta I^{0}+(m+d) & -d & (1-\theta) \alpha I^{0} \\
0 & \lambda & 0 & -k
\end{array}\right) .
$$

With regard to this matrix, the eigenvalues are the roots of the polynomial equation

$$
\begin{aligned}
& {\left[x^{2}+\left(m+d+k-\theta \beta I^{0}\right) x+\left(m+d-\theta \beta I^{0}\right) k-\lambda \theta \alpha I^{0}\right]} \\
& \quad \times(x+d)(x+k)=0 .
\end{aligned}
$$

It is easy to know that $-d$ and $-k$ are two of the eigenvalues. When $R_{0}>1,\left(m+d-\theta \beta I^{0}\right) k-\lambda \theta \alpha I^{0}<0$; that is, there must exist a positive root. That means that $E_{0}$ is unstable.

With regard to the positive equilibrium $E^{*}=\left(I^{*}, S^{*}, R^{*}\right.$, $\left.W^{*}\right)$, it should satisfy

$$
\begin{gathered}
A-\beta I^{*} S^{*}-\alpha I^{*} W^{*}-d I^{*}=0, \\
\theta \beta I^{*} S^{*}+\theta \alpha I^{*} W^{*}-2 \xi_{1} S^{* 2}-m S^{*}-\xi_{2} S^{*} R^{*}-d S^{*}=0, \\
(1-\theta) \beta I^{*} S^{*}+(1-\theta) \alpha I^{*} W^{*}+2 \xi_{1} S^{* 2}+m S^{*} \\
+\xi_{2} S^{*} R^{*}-d R^{*}=0, \\
\lambda S^{*}-k W^{*}=0 .
\end{gathered}
$$

By calculating the equations, we have

$$
x_{1} S^{2}+x_{2} S+x_{3}=0 \text {, }
$$

where

$$
\begin{aligned}
& x_{1}=\left(\xi_{2}-2 \xi_{1}\right)(k \beta+\alpha \lambda), \\
& x_{2}=\left(\xi_{2}-2 \xi_{1}\right) k d-\left(m+d+\xi_{2} \frac{A}{d}\right)(k \beta+\alpha \lambda), \\
& x_{3}=\theta \beta A k+\theta \alpha \lambda A-(m+d) k d=k(m+d)\left(R_{0}-1\right) .
\end{aligned}
$$

The analysis about $x_{1}, x_{2}$, and $x_{3}$ is more complex and we list the result in Table 2 .

\section{A Dynamical System for Rumor Spreading with Government Measures}

Now, we add the measures of government to the system, especially issuing the actual message through the medium $G(t)$ and punishment for the spreaders, which are reflected in $\eta$ and $\mu$. Moreover, the ability of cognizance of the public is reflected in $\theta$. The higher the cognizance ability the smaller the $\theta$. These interpretations can be seen in Table 2. $B(S)$ can be adopted as different term according to different situation. The system has the following form:

$$
\begin{gathered}
\frac{d I(t)}{d t}=A-\beta I(t) S(t)-\alpha I(t) W(t)-\eta \gamma I(t) G(t) \\
\quad-d I(t) \\
\frac{d S(t)}{d t}=\theta \beta I(t) S(t)+\theta \alpha I(t) W(t)-2 \xi_{1} S^{2}(t)-m S(t) \\
\quad-\xi_{2} S(t) R(t)-\eta \delta S(t) G(t)-d S(t)-\mu S(t), \\
\frac{d R(t)}{d t}=(1-\theta) \beta I(t) S(t)+(1-\theta) \alpha I(t) W(t)+2 \xi_{1} S^{2}(t) \\
+m S(t)+\mu S(t)+\xi_{2} S(t) R(t)+\eta \delta S(t) G(t) \\
+\eta \gamma I(t) G(t)-d R(t), \\
\frac{d W(t)}{d t}=\lambda S(t)-k W(t) \\
\frac{d G(t)}{d t}=B(S)-p G(t) .
\end{gathered}
$$

\section{Dynamical Behavior of System (10)}

What this paper mainly discusses is the effect of measures carried out by authority. At first, we can assume that the 


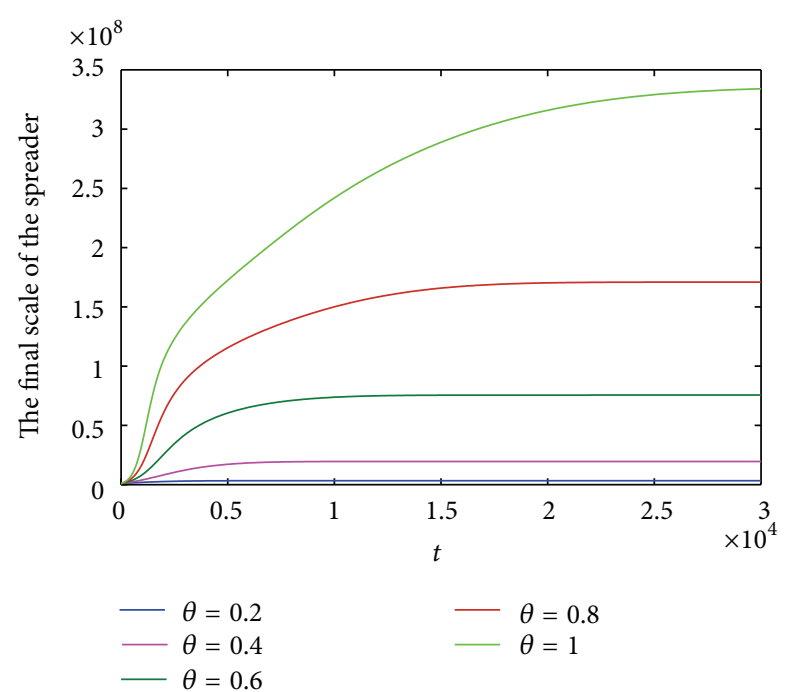

(a)
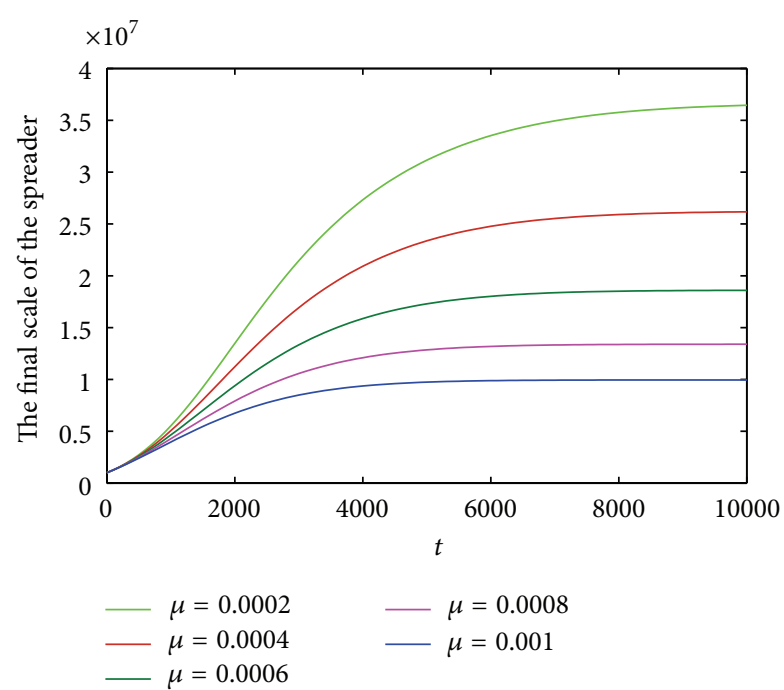

(c)

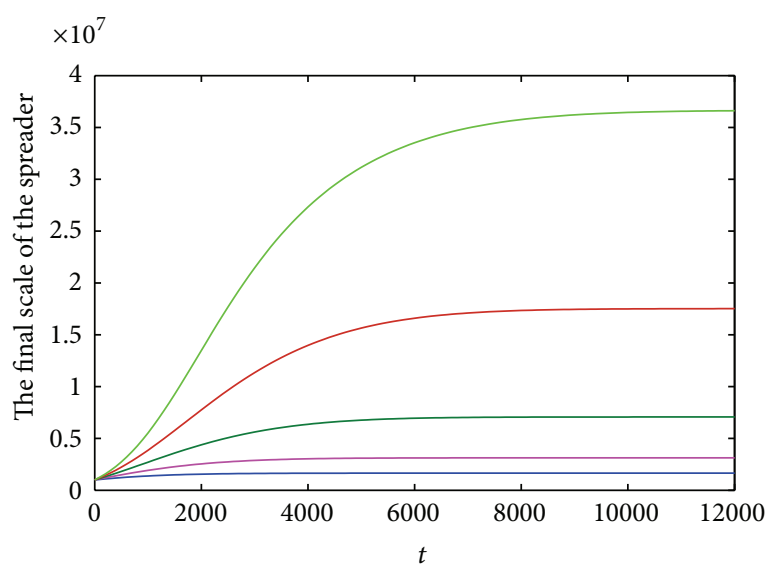
$-\beta=50 \times 0.0002 /\left(1.35 \times 10^{9}\right)-\beta=50 \times 0.0008 /\left(1.35 \times 10^{9}\right)$
$-\beta=50 \times 0.0004 /\left(1.35 \times 10^{9}\right)-\beta=50 \times 0.001 /\left(1.35 \times 10^{9}\right)$
$\beta=50 \times 0.0006 /\left(1.35 \times 10^{9}\right)$

(e)

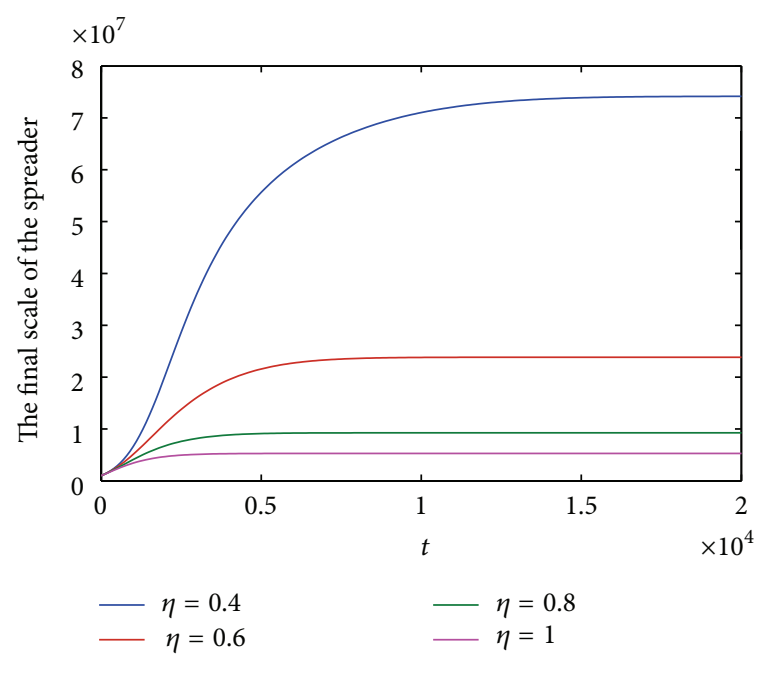

(b)

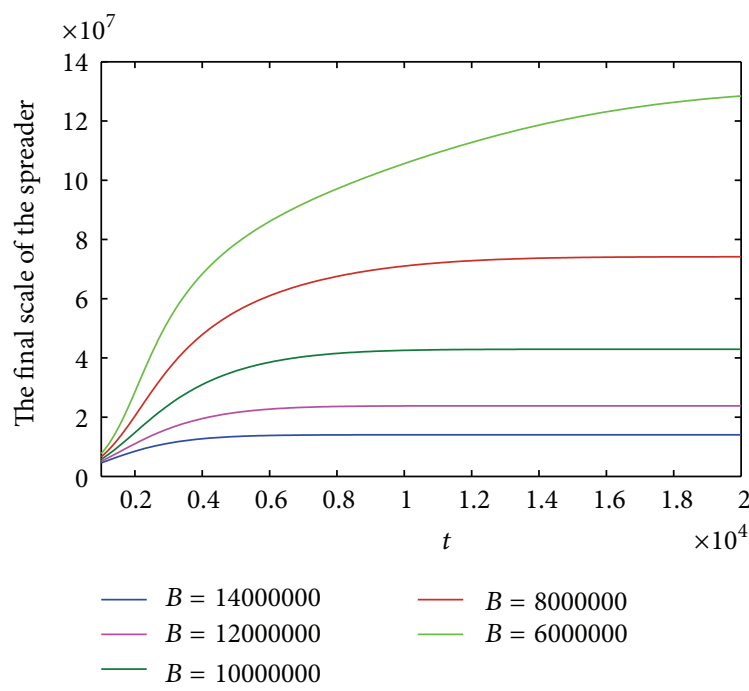

(d)

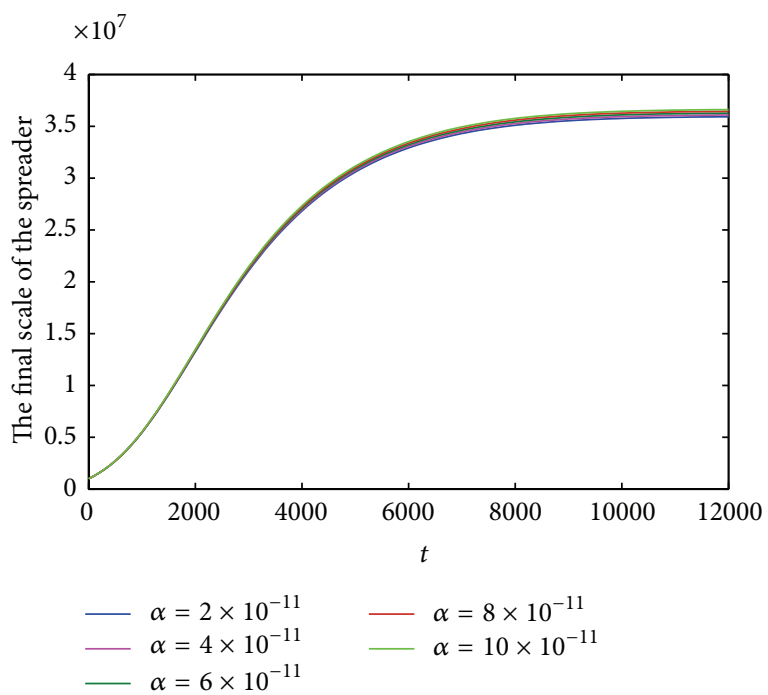

(f)

FIGURE 2: Continued. 


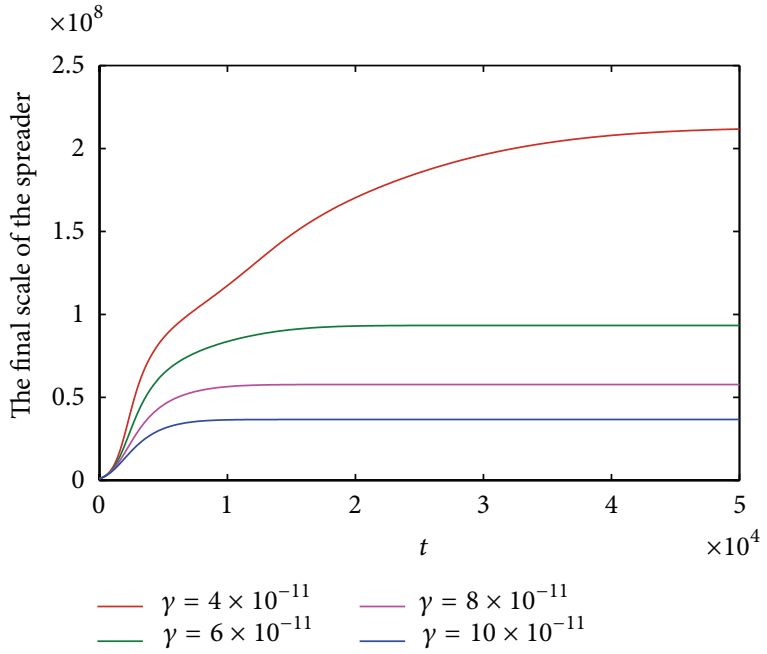

(g)

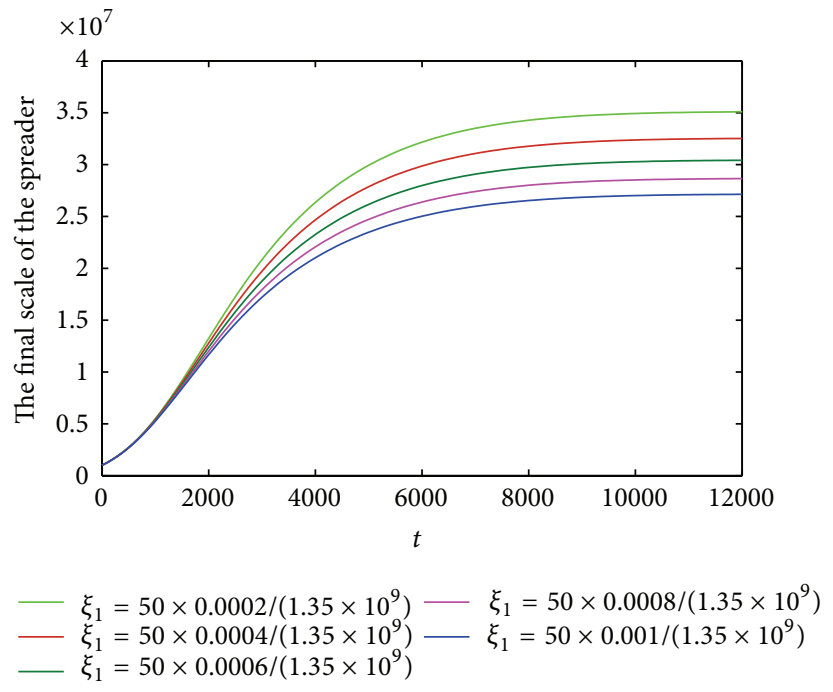

(h)

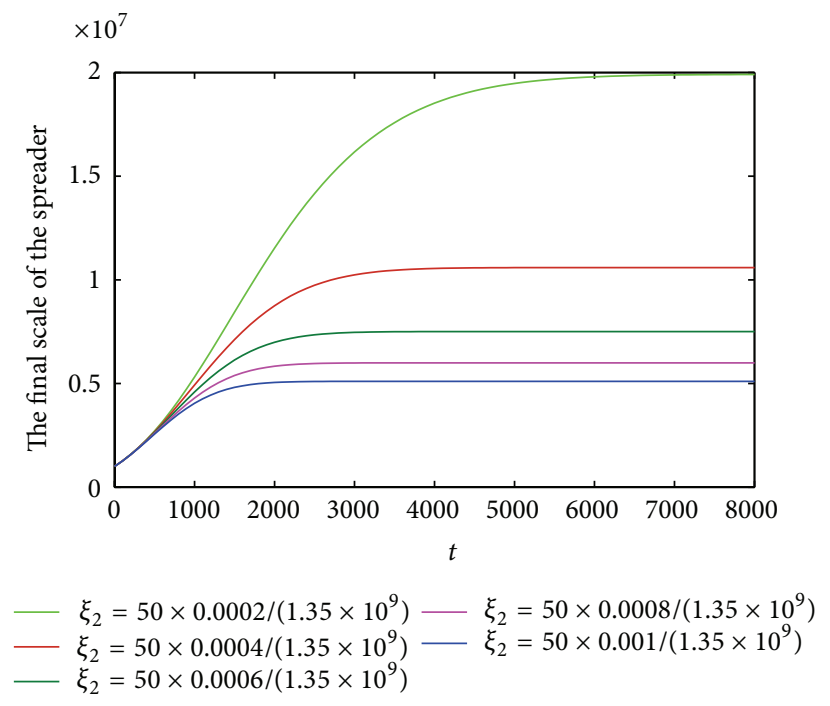

(i)

FIgURE 2: The sensitivity of the final scale in terms of parameters. (a) $\theta$. (b) $\eta$. (c) $\mu$. (d) $B$. (e) $\beta$. (f) $\alpha$. (g) $\gamma$. (h) $\xi_{1}$. (i) $\xi_{2}$.

TABLE 2: Equilibria and stability of system (1).

\begin{tabular}{lccc}
\hline Cases & & Conditions & Positive equilibria \\
\hline \multirow{2}{*}{$R_{0}<1$} & $\xi_{2}<2 \xi_{1}$ & $x_{1}<0, x_{2}<0, x_{3}<0$ & No positive equilibrium \\
& $\xi_{2}=2 \xi_{1}$ & $x_{1}=0, x_{2}<0, x_{3}<0$ & No positive equilibrium \\
& $\xi_{2}>2 \xi_{1}$ & $x_{1}>0, x_{3}<0$ & Unique positive equilibrium $E^{*}$ \\
\hline$\xi_{2}<2 \xi_{1}$ & $x_{1}<0, x_{3}>0$ & Unique positive equilibrium $E^{*}$ \\
& $\xi_{2}=2 \xi_{1}$ & $x_{1}=0, x_{2}<0, x_{3}>0$ & Unique positive equilibrium \\
& & $x_{2} \geq 0, x_{1}>0, x_{3}>0$ & No positive equilibrium \\
$R_{0}>1$ & $\xi_{2}>2 \xi_{1}$ & $x_{2}<0, x_{1}>0, x_{3}>0, \Delta<0$ & No positive equilibrium \\
& & $x_{2}<0, x_{1}>0, x_{3}>0, \Delta=0$ & Unique positive equilibrium \\
& & $x_{2}<0, x_{1}>0, x_{3}>0, \Delta>0$ & Two positive equilibria \\
\hline
\end{tabular}




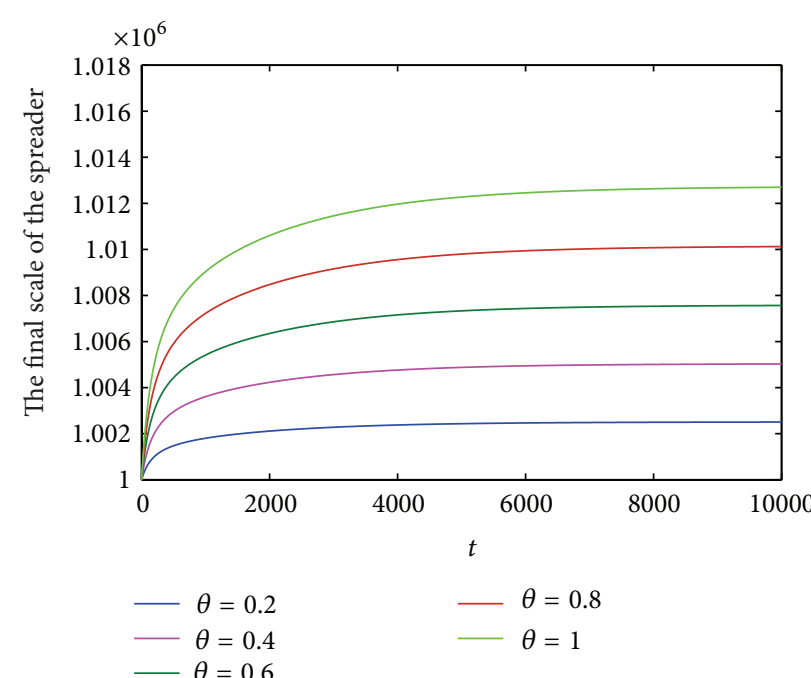

(a)
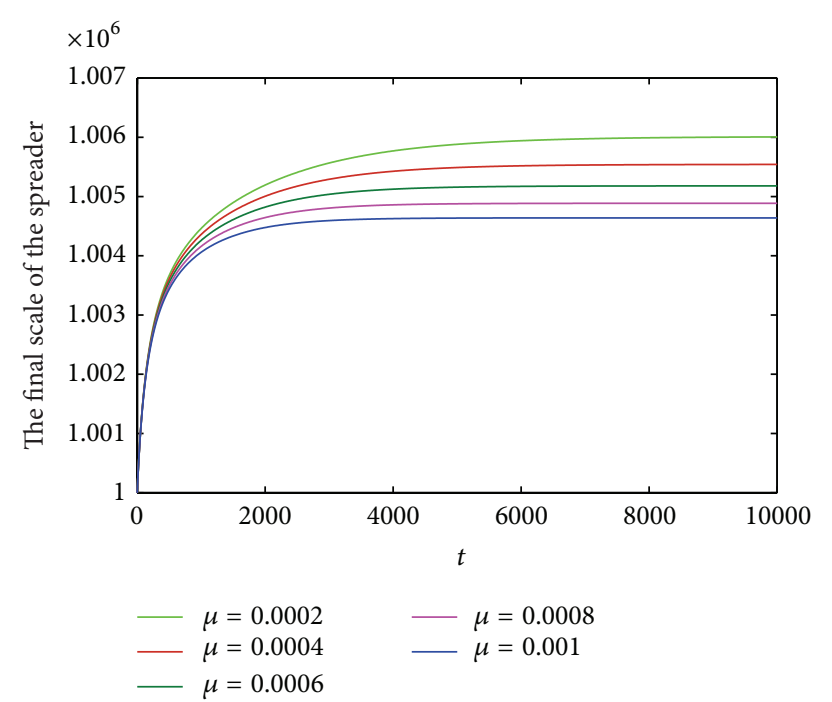

(c)

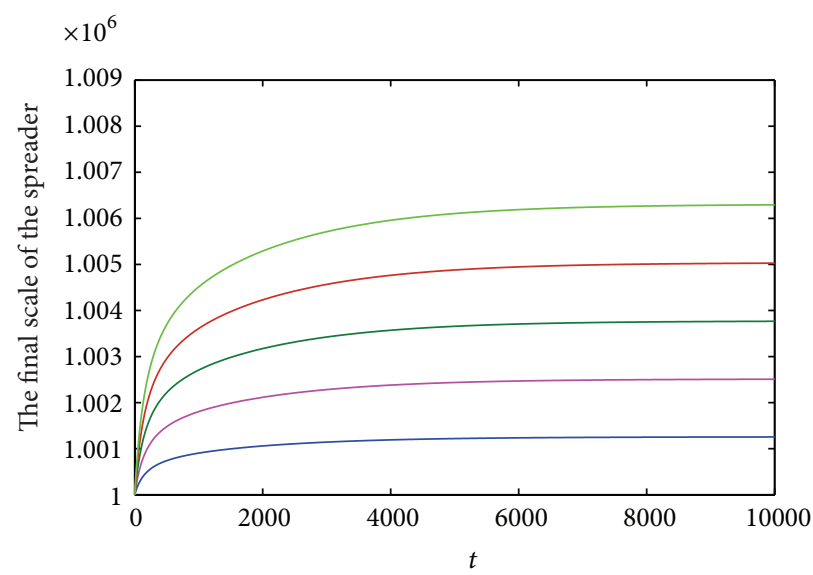

\footnotetext{
$-\beta=50 \times 0.000002 /\left(1.35 \times 10^{9}\right)-\beta=50 \times 0.000008 /\left(1.35 \times 10^{9}\right)$

$-\beta=50 \times 0.000004 /\left(1.35 \times 10^{9}\right)-\beta=50 \times 0.00001 /\left(1.35 \times 10^{9}\right)$

$-\beta=50 \times 0.000006 /\left(1.35 \times 10^{9}\right)$
}

(e)

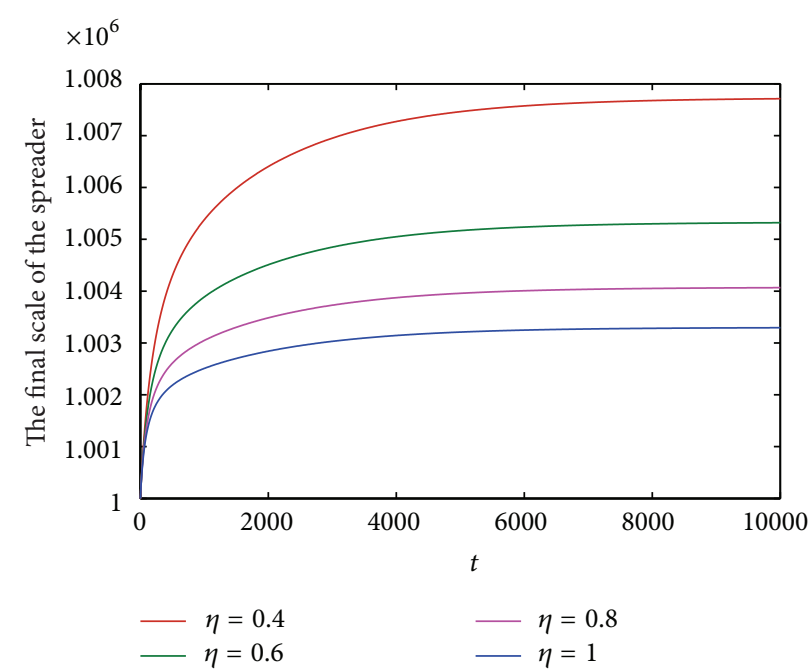

(b)

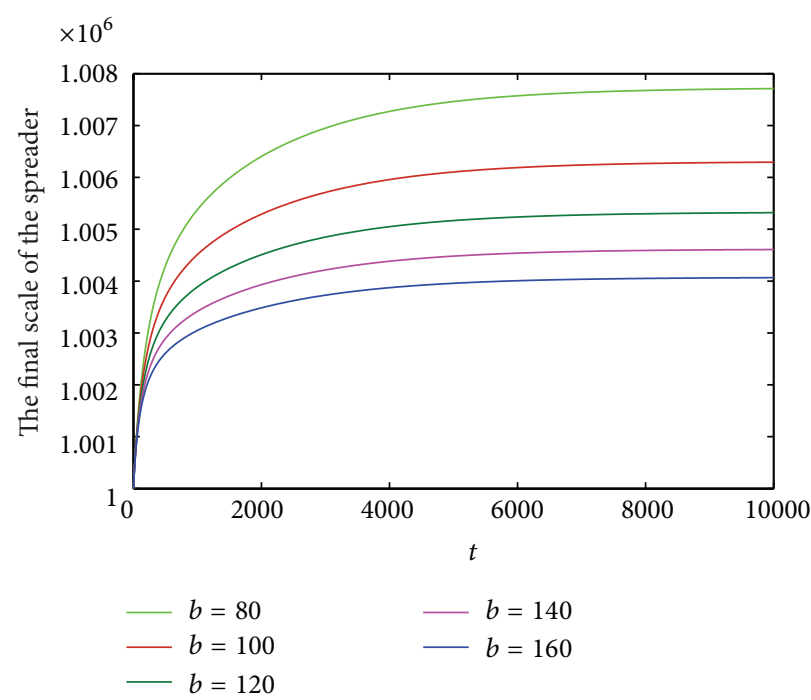

(d)

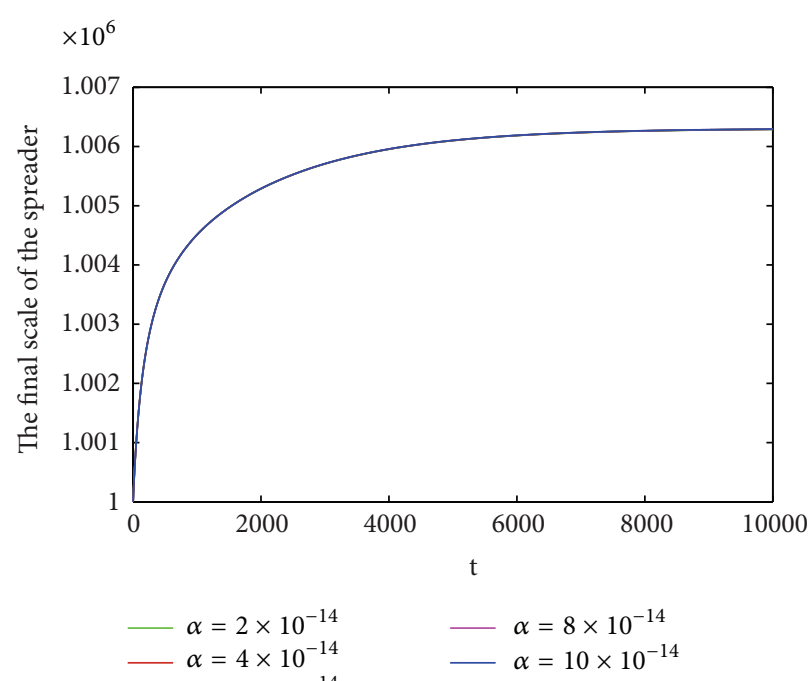

(f)

FIgUre 3: Continued. 


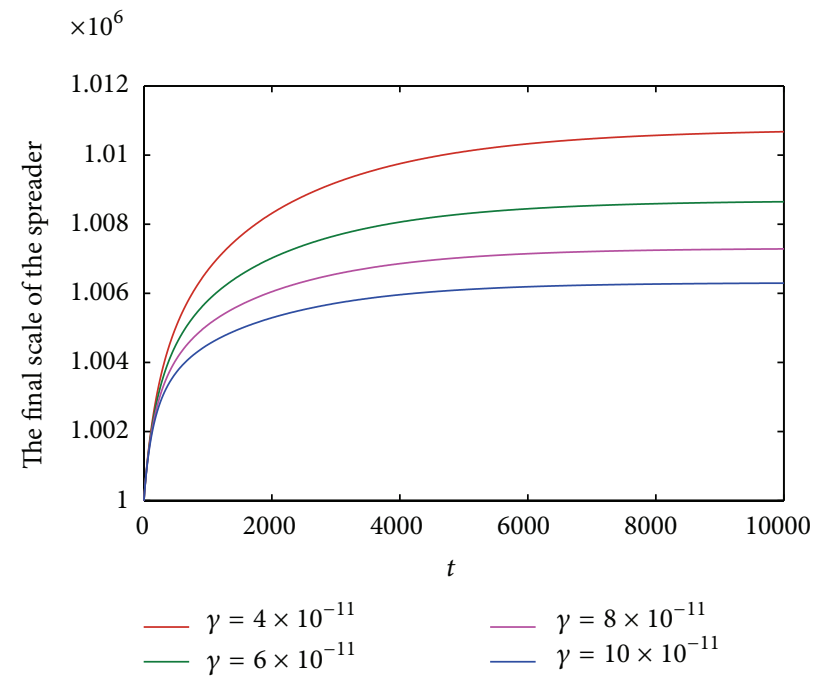

(g)

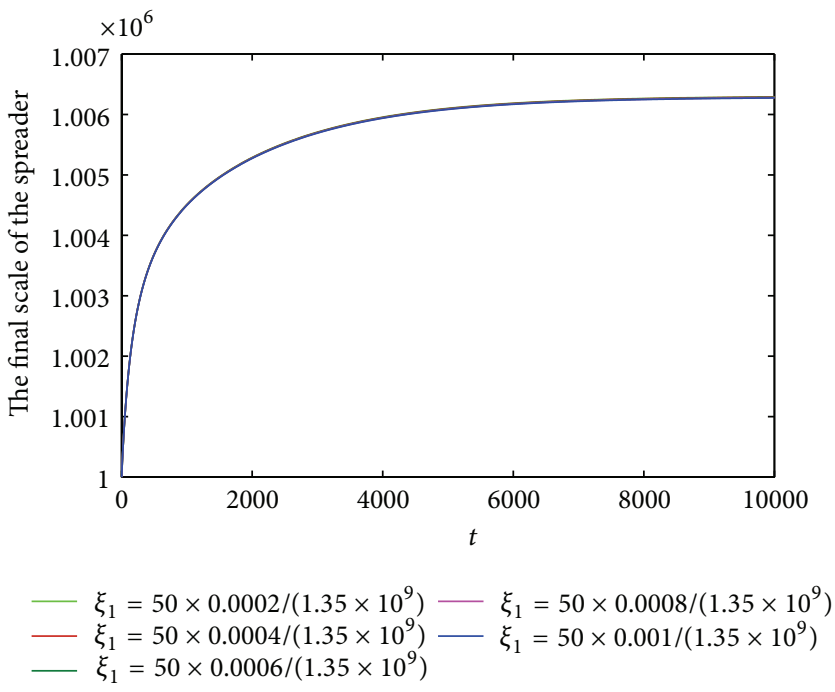

(h)

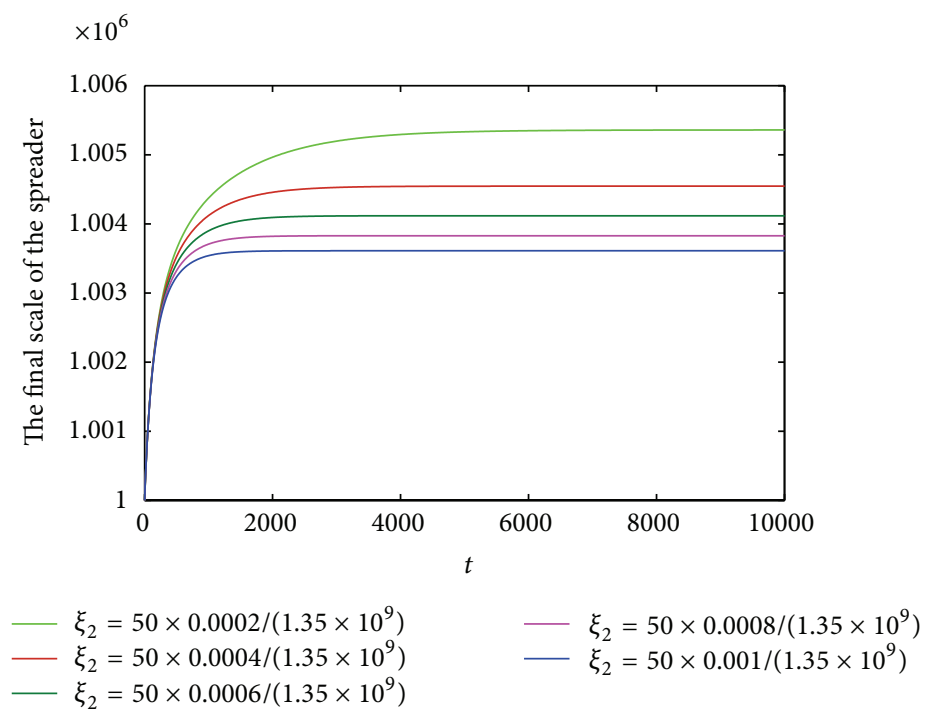

(i)

FiguRE 3: The sensitivity of the final scale in terms of parameters. (a) $\theta$. (b) $\eta$. (c) $\mu$. (d) $b$. (e) $\beta$. (f) $\alpha$. (g) $\gamma$. (h) $\xi_{1}$. (i) $\xi_{2}$.

TABLE 3: Description of parameters in the system (10).

\begin{tabular}{lccc}
\hline Parameters & Value & Unit & Comments \\
\hline$\beta$ & $50 \times 0.00001 / 1350000000$ & Day $^{-1}$ & $S(t)$-to- $I(t)$ transmission rate \\
$\alpha$ & $10^{-13}$ & Day $^{-1}$ & $W(t)$-to- $I(t)$ transmission rate \\
$\eta$ & 0.5 & Day $^{-1}$ & The rate to become $R(t)$ after receiving $G(t)$ \\
$\gamma$ & $10^{-10}$ & Day $^{-1}$ & $G(t)$-to- $S(t)$ transmission rate \\
$\mu$ & 0.0001 & Day $^{-1}$ & The punishment rate for the spreaders \\
$B$ & 1000000 & Day $^{-1}$ & The quantity of messages by government \\
$b$ & 100 & Day $^{-1}$ & The quantity of messages by government \\
$p$ & 1 & Day $^{-1}$ & The submerged rate of messages by government \\
$G(0)$ & 0 & Individual $^{-1}$ & The initial quantity of messages by government \\
\hline
\end{tabular}


authority will release quantitative trustworthy message per time. So, the behavior of authority is independent of the rumor spreading; that is, $B(S)=B$. The parameters of system (10) are in Table 3.

Case $1(B(S)=B)$. In this case, the disease-free equilibrium $E_{01}=\left(I^{0}, 0, R^{0}, 0, G^{0}\right)$, where $I^{0}=A p /(\eta B r+d p), R^{0}=$ $\eta r A B /(\eta B r+d p) d, G^{0}=B / p$. Let us look at the basic reproduction number of the spreading of rumor. For the rumor spread, one has

$$
\begin{gathered}
\mathscr{F}=\left(\begin{array}{c}
\theta \beta I S+\theta \alpha I W \\
0 \\
0
\end{array}\right), \\
\mathscr{V}=\left(\begin{array}{c}
\eta \delta S G+2 \xi_{1} S^{2}+m S+\xi_{2} S R+d S+\mu S \\
k W-\lambda S \\
p G-B
\end{array}\right) .
\end{gathered}
$$

So, we derive

$$
\begin{gathered}
F(t)=\left(\begin{array}{ccc}
\theta \beta I^{0} & \theta \alpha I^{0} & 0 \\
0 & 0 & 0 \\
0 & 0 & 0
\end{array}\right), \\
V(t)=\left(\begin{array}{cccc}
\eta \delta G^{0}+\xi_{2} R^{0}+m+d+\mu & 0 & 0 \\
-\lambda & k & 0 \\
0 & 0 & p
\end{array}\right) .
\end{gathered}
$$

Then, the basic reproduction number is $R_{01}=\rho\left(F V^{-1}\right)=$ $\theta \beta I^{0} /\left(m+d+\mu+\xi_{2} R^{0}+\eta \delta G^{0}\right)+\lambda \theta \alpha I^{0} /\left(m+d+\mu+\xi_{2} R^{0}+\right.$ $\left.\eta \delta G^{0}\right) k$. From expression of $R_{01}$, we can see that the measures of government reduce the basic reproduction number.

Under some situations, once the rumor emerges, the government will issue the news to clarify the rumor and the message released by the authority. The more the spreaders there are the more the message the authority should issue. Thus, we can let $B(S)=b S^{n}, n \in \mathbb{N}^{+}$.

Case $2\left(B(S)=b S^{n}, n \in \mathbb{N}^{+}\right)$. In Case 2, the diseasefree equilibrium $E_{0}=(A / d, 0,0,0,0)$. Similarly, the basic reproduction number is $R_{02}=\rho\left(F V^{-1}\right)=\theta \beta I^{0} /(m+d+\mu)+$ $\lambda \theta \alpha I^{0} /(m+d+\mu) k$, which does not relate to the parameters $\eta, b$. From the expression of $R_{02}$, we can know that $R_{02}$ is the same as $R_{0}$. Thus, if the government adopts measure after the appearance of rumor during the early stage of the rumor spread, the measure cannot change the value of the basic reproduction number.

Case $3\left(B(S)=B+b S^{n}, n \in \mathbb{N}^{+}\right)$. In this case, the disease-free equilibrium $E_{01}=\left(I^{0}, 0, R^{0}, 0, G^{0}\right)$, where $I^{0}=A p /(\eta B r+$ $d p), R^{0}=\eta r A B /((\eta B r+d p) d), G^{0}=B / p$. And the basic reproduction number is $R_{01}=\rho\left(F V^{-1}\right)=\theta \beta I^{0} /(m+d+\mu+$ $\left.\xi_{2} R^{0}+\eta \delta G^{0}\right)+\lambda \theta \alpha I^{0} /\left(m+d+\mu+\xi_{2} R^{0}+\eta \delta G^{0}\right) k$, which is similar to Case 1 .

\section{Sensitivity Analysis}

This paper mainly discusses the effect of measures adopted by government. On the one hand, in the early stage, the sensitivity of the basic reproduction numbers about parameters that correspond to measures adopted by government should be discussed. On the other hand, when $R_{01}<1$ or $R_{0}<1$, the sensitivity of the final scale of the spreader about parameters should be studied.

Now, we carry out the sensitivity analysis under different cases.

Case $1(B(S)=B)$. Consider

$$
\begin{aligned}
R_{01} & =\rho\left(F V^{-1}\right) \\
& =\frac{\theta \beta I^{0}}{m+d+\mu+\xi_{2} R^{0}+\eta \delta G^{0}}+\frac{\lambda \theta \alpha I^{0}}{\left(m+d+\mu+\xi_{2} R^{0}+\eta \delta G^{0}\right) k} .
\end{aligned}
$$

Observing Figure $1, R_{01}$ is linear function of $\theta, \lambda$, and $p$. $R_{01}$ is the concave function with the rest of parameters, where the influences of $\eta, B$, and $\mu$ are greater on $R_{01}$. Observing the values of ordinate axis, $B$ and $\lambda$ have the biggest influence on $R_{01}$; that is, the releasing amount of messages is the most important. With regard to $k$ and $p$, the submerged rate of message issued by the government has bigger effect than $k$. So, for message by spreader, we should control the distribution of message. Once the message is issued, the deleting of message has a small effect on controlling rumor spreading. For the government, in order to prevent the rumor spread, the quantity and the survival time of message are important factors. From $\eta$ and $\theta$, the reliability of government and the cognizance ability of the public are equally important and $R_{01}$ is more sensitive with the reliability of government. With regard to $\mu$, the concavity of curve is the biggest. When the minority of people are published, the effect has been big on $R_{01}$. From Figures $1(\mathrm{~h}), 1(\mathrm{i})$, and $1(\mathrm{j})$, it is easy to know that $\gamma$ has the biggest effect on $R_{01}$. The effect of $\alpha$ on $R_{01}$ is the smallest.

When $R_{01}<1$, as time goes on, the rumor will eventually disappear. In this case, what we should focus on is the final scale of the spreader. Next, we discuss the influences of parameters on the final scale.

From Figures 2(a) and 2(b), we can see that $\eta$ has a bigger influence on the final scale than $\theta$, which means that the reliability of government is more important. Comparing Figures 2(c) and 2(d), the change of the final scale caused by the $B$ is bigger than $\mu$, which implies that the effect of release of message is more obvious than punishment from government. From Figures 2(e), 2(f), and 2(g), we know that when $R_{01}<1$, the influence of $\alpha$ is smaller. For $\gamma, \xi_{1}$, and $\xi_{2}$, the influence of $\gamma$ is the biggest and is followed by $\xi_{2}$ and $\xi_{1}$.

Case $2(B(S)=b S)$. The basic reproduction number of the whole system is $R_{0}=\theta \beta I^{0} /(m+d+\mu)+\lambda \theta \alpha I^{0} /(m+d+\mu) k$. We can know that the basic reproduction number does not 
change. For such reason, we should focus on the final scale of the spreader, which is showed in Figure 3.

When $B(S)=b S$, then $\theta$ has a bigger influence on the final scale than $\eta$, which is different from Case 1. Observing Figure 2(c), the effect of $\mu$ is smaller. The changes of the final scales of the spreader are very little under the changes of $\alpha$ and $\xi_{1}$. The effect of $\beta$ is larger than $\xi_{2}$ and $\gamma$.

\section{Discussion}

Applying the dynamical system, this paper describes the government measures by the parameters $\eta, \theta, \mu, \lambda, k, B$, $b$, and $p$. More specifically, $\eta$ indicates the reliability of government, $\theta$ indicates the ability of cognizance of the public, $\mu$ indicates the punishment rate of the government, $k$ reflects the management strength of government for the internet, $B$ and $b$ show the amount of messages released by authority, $\beta$ is the transmission rate between humans directly, $\alpha$ is the transmission rate of media to human, and $\gamma$ is the transmission rate of the government to human by issuing presentation.

According to the above dynamical analysis and sensitivity analysis, we can know that $B$ and $b$ have the greatest influence on the rumor spread. The effects of $\eta$ and $\theta$ are almost big. When $B(S)=B$, the influence of $\eta$ is larger which means that the reliability of government is more important when the government issues message beforehand. When $B(S)=b S$, the influence of $\theta$ is larger which means that the cognizance ability of the public is more important when the government releases the message according to the number of the spreaders. The effects of $k, \lambda$, and $p$ explain that monitoring the internet to prevent the diffusion of rumor is more important than the deleting message in media that has appeared. Moreover, extending the survival time of government message is also necessary. The relationship of $R_{01}$ in terms of $\mu$ shows that when government punishes the minority of people, the effect is obvious. However, with the increase of $\mu$, the effect is weakened. $R_{01}$ is a concave function with $\gamma$. From Figure 1(j), we can know that the influence of $\gamma$ is larger than $\beta$ and $\alpha$.

In [26], an ISRW model was presented and its dynamical behaviors were well investigated. Reference [26] was mainly based on the Jacobian matrix and obtained the final size of rumor. However, this paper is based on spectral radius and focuses on the effects of different measures. The obtained results well will enrich the findings in rumor spreading.

\section{Conflict of Interests}

The authors declare that there is no conflict of interests regarding the publication of this paper.

\section{References}

[1] S. C. Pendleton, "Rumor research revisited and expanded," Language \& Communication, vol. 1, no. 18, pp. 69-86, 2008.

[2] S. Young, A. Pinkerton, and K. Dodds, "The word on the street: rumor, "race" and the anticipation of urban unrest," Political Geography, vol. 38, pp. 57-67, 2014.
[3] W. Li, S. Tang, S. Pei et al., "The rumor diffusion process with emerging independent spreaders in complex networks," Physica A, vol. 397, pp. 121-128, 2014.

[4] J. J. Wang, L. J. Zhao, and R. B. Huang, "SIRaRu rumor spreading model in complex networks," Physica A, vol. 398, pp. 43-55, 2014.

[5] S. Han, F. Z. Zhuang, Q. He, Z. Z. Shi, and X. Ao, "Energy model for rumor propagation on social networks," Physica A, vol. 394, pp. 99-109, 2014.

[6] Y. L. Zan, J. L. Wu, P. Li, and Q. L. Yu, "SICR rumor spreading model in complex networks: counterattack and self-resistance," Physica A, vol. 405, pp. 159-170, 2014.

[7] D. J. Daley and D. G. Kendall, "Epidemics and rumours," Nature, vol. 204, pp. 11-18, S1464-S3634, 1964.

[8] D. P. Maki and M. Thompson, Mathematical Models and Applications, Prentice Hall, Englewood Cliffs, NJ, USA, 1973.

[9] L. J. Zhao, J. J. Wang, Y. C. Chen et al., "SIHR rumor spreading model in social networks," Physica A, vol. 391, pp. 2444-2453, 2012.

[10] K. Kawachi, M. Seki, H. Yoshida, Y. Otake, K. Warashina, and H. Ueda, "A rumor transmission model with various contact interactions," Journal of Theoretical Biology, vol. 253, no. 1, pp. 55-60, 2008.

[11] L. J. Zhao, H. X. Cui, X. Y. Qiu, X. L. Wang, and J. J. Wang, "SIR rumor spreading model in the new media age," Physica A, vol. 392, no. 4, pp. 995-1003, 2013.

[12] L. Huo, T. T. Lin, and P. Q. Huang, "Dynamical behavior of a rumor transmission model with psychological effect in emergency event," Abstract and Applied Analysis, vol. 2013, Article ID 282394, 9 pages, 2013.

[13] G. H. Chen, H. Z. Shen, T. Ye, G. M. Chen, and N. Kerr, "A kinetic model for the spread of rumor in emergencies," Discrete Dynamics in Nature and Society, vol. 2013, Article ID 605854, 8 pages, 2013.

[14] Y. Naimi and M. Naimi, "Reliability and efficiency of generalized rumor spreading model on complex social networks," Communications in Theoretical Physics, vol. 60, pp. 139-144, 2013.

[15] J. Y. Huang and X. G. Jin, "Preventing rumor spreading on small-world networks," Journal of Systems Science \& Complexity, vol. 24, no. 3, pp. 449-456, 2011.

[16] D. H. Zanette, "Dynamics of rumor propagation on small-world networks," Physical Review E, vol. 65, Article ID 041908, 2002.

[17] Y. Moreno, M. Nekovee, and A. F. Pacheco, "Dynamics of rumor spreading in complex networks," Physical Review E, vol. 69, Article ID 066130, 2004.

[18] D. Trpevski, "Model for rumor spreading over networks," Physical Review E, vol. 81, Article ID 056102, 2010.

[19] Y. C. Zhang, S. Zhou, Z. Z. Zhang, J. H. Guan, and S. G. Zhou, "Rumor evolution in social networks," Physical Review E, vol. 87, Article ID 032133, 2013.

[20] B. Doerr, A. Huber, and A. Levavi, "Strong robustness of randomized rumor spreading protocols," Discrete Applied Mathematics, vol. 161, no. 6, pp. 778-793, 2013.

[21] E. Lebensztayn and P. M. Rodriguez, "A connection between a system of random walks and rumor transmission," Physica A, vol. 392, no. 23, pp. 5793-5800, 2013.

[22] N. Fountoulakis and K. Panagiotou, "Rumor spreading on random regular graphs and expanders," Random Structures and Algorithms, vol. 43, no. 2, pp. 201-220, 2013. 
[23] C. F. Coletti, P. M. Rodríguez, and R. B. Schinazi, "A spatial stochastic model for rumor transmission," Journal of Statistical Physics, vol. 147, no. 2, pp. 375-381, 2012.

[24] Y. Zhang and Y. Guo, "A study on rumor propagation model on micro-blogging platforms," Advanced Materials Research, vol. 717, pp. 808-811, 2013.

[25] L. Ma, Z. T. Jia, H. Y. Sun, and C. Yu, "An improved model of the internet public opinion spreading on mass emergencies," Applied Mechanics and Materials, vol. 433-435, pp. 1760-1764, 2013.

[26] X. Zhao and J. Wang, "Dynamical model about rumor spreading with medium," Discrete Dynamics in Nature and Society, vol. 2013, Article ID 586867, 9 pages, 2013.

[27] L. A. Huo, P. Q. Huang, and X. Fang, "An interplay model for authorities' actions and rumor spreading in emergency event," Physica A, vol. 390, no. 20, pp. 3267-3274, 2011.

[28] Y. Y. Bao, Y. Niu, C. Q. Yi, and Y. B. Xue, "Effective immunization strategy for rumor propagation based on maximum spanning tree," in Proceedings of the 2014 Interernational Conference on Computing, Networking and Communications, Communications and Information Security Symposium, 2014.

[29] K. H. Ji, J. W. Liu, and G. Xiang, "Anti-rumordynamics and rumor control strategies on complex network," http://arxiv.org/ abs/1310.7198.

[30] H. Rahmandad and J. Sterman, "Heterogeneity and network structure the dynamics of diffusion: comparing agent-based and differential equation models," Management Science, vol. 54, pp. 998-1014, 2008.

[31] L. V. Green and P. J. Kolesar, "Improving emergency responsiveness with improving emergency responsiveness with management science," Management Science, vol. 50, pp. 1001-1014, 2004.

[32] X.-X. Zhao and J.-Z. Wang, "Dynamics of an information spreading model with isolation," Abstract and Applied Analysis, vol. 2014, Article ID 484630, 6 pages, 2014.

[33] R. M. Anderson and R. M. May, Infectious Diseases of Humans, Oxford University Press, Oxford, UK, 1991.

[34] O. Diekmann and J. A. P. Heesterbeek, Mathematical Epidemiology of Infectious Diseases, Wiley Series in Mathematical and Computational Biology, John Wiley \& Sons, West Sussex, UK, 2000.

[35] P. van den Driessche and J. Watmough, "Reproduction numbers and sub-threshold endemic equilibria for compartmental models of disease transmission," Mathematical Biosciences, vol. 180, pp. 29-48, 2002.

[36] J. LaSalle and S. Lefschetz, Stability by Liapunov's Direct Method, Academic Press, New York, NY, USA, 1961.

[37] E. A. Barbashin, Introduction to the Theory of Stability, WoltersNoordhoff, Groningen, The Netherlands, 1970. 


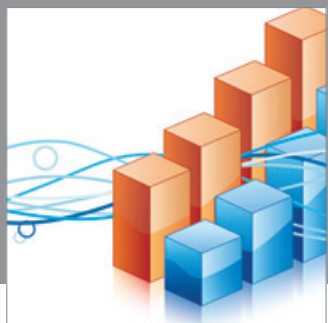

Advances in

Operations Research

mansans

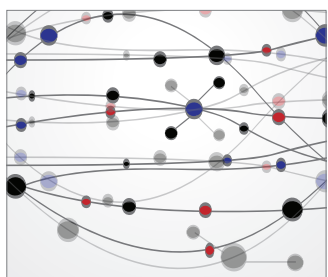

The Scientific World Journal
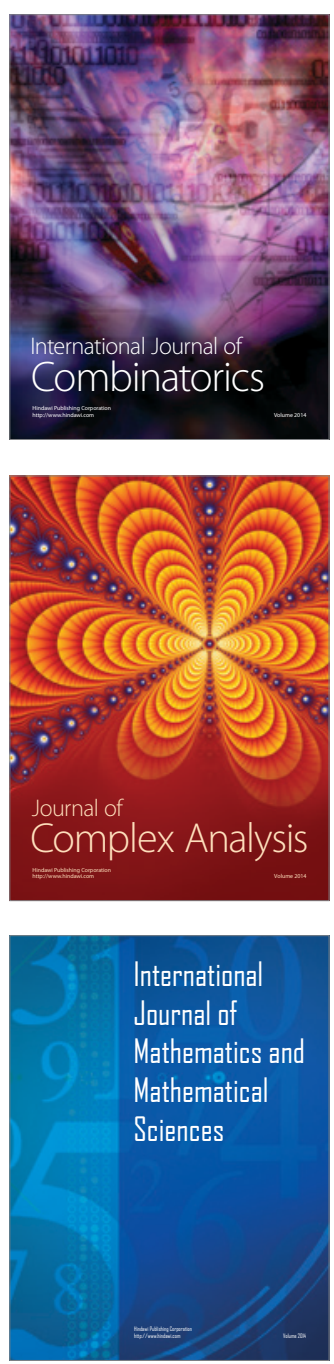
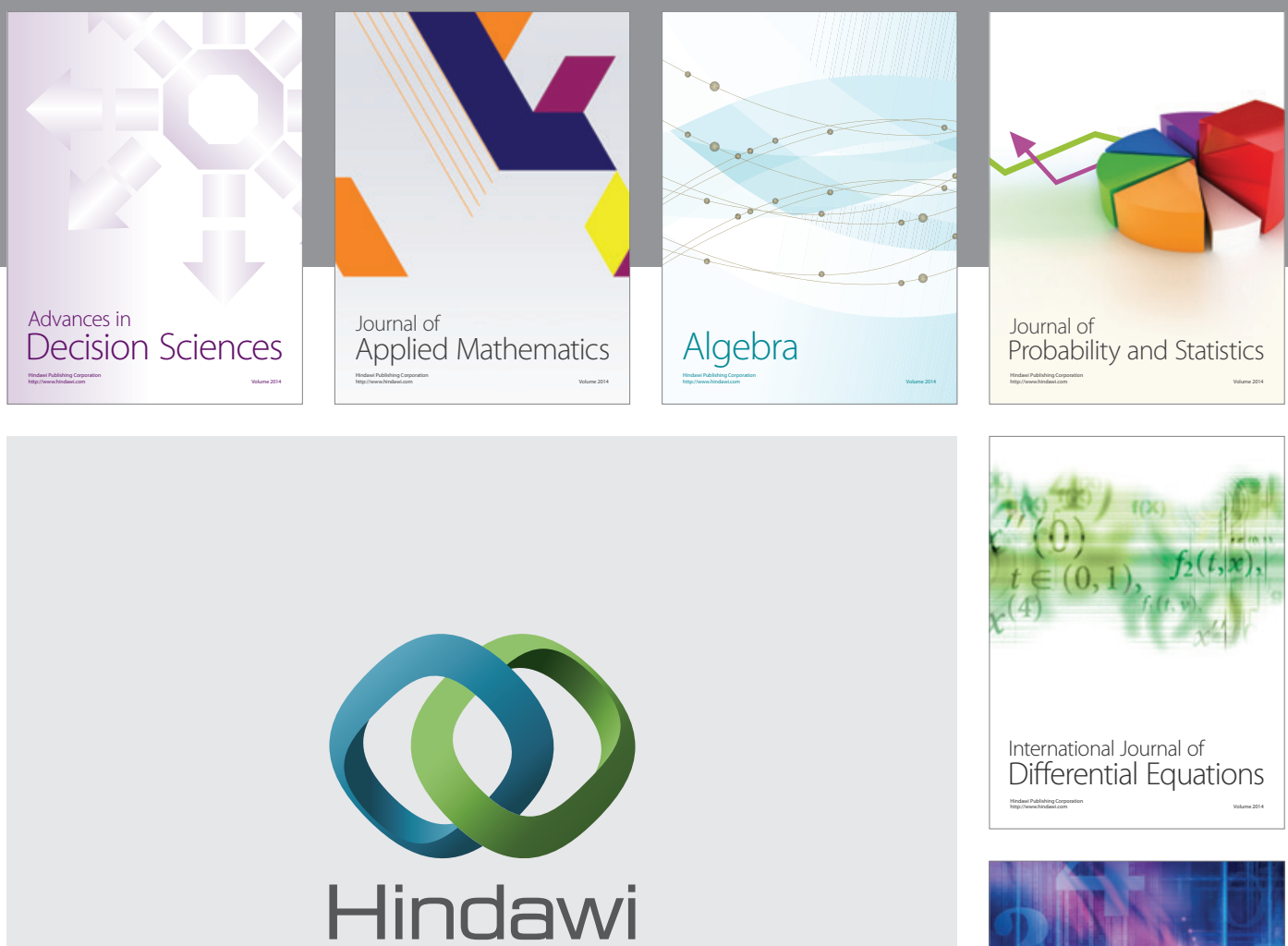

Submit your manuscripts at http://www.hindawi.com
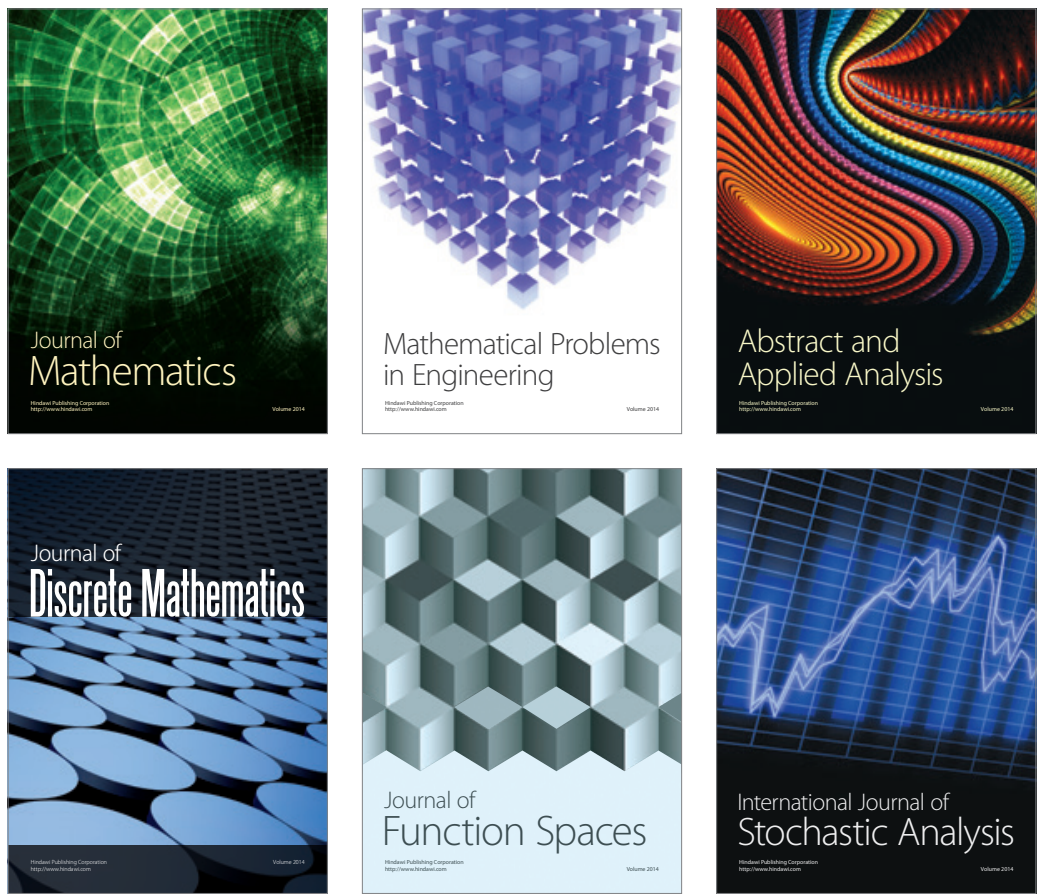

Journal of

Function Spaces

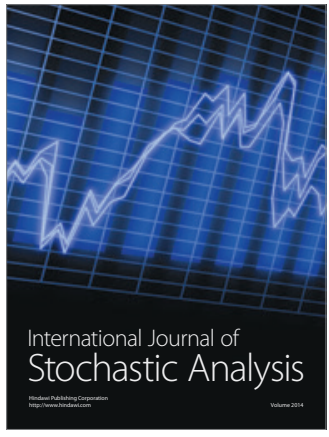

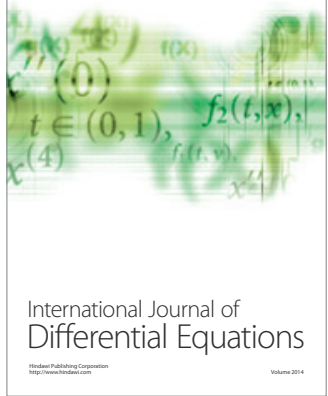
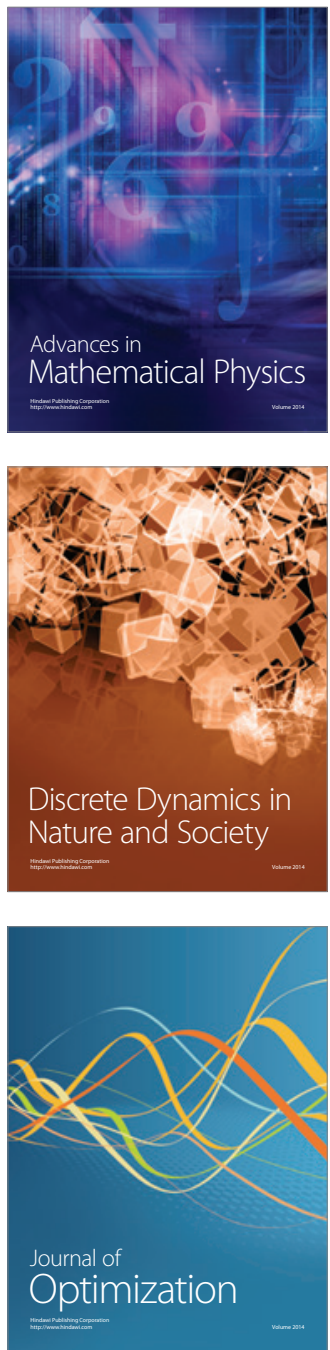